\title{
The interfacial profile in two-loop order
}

\author{
Jens Küster and Gernot Münster*
}

October 13, 2006

(revised: July 27, 2007)

\begin{abstract}
The profile of interfaces separating different phases of statistical systems is investigated in the framework of renormalized field theory. The profile function is calculated analytically in the local potential approximation, using the effective potential to two loops. It can be interpreted as an intrinsic interfacial profile. The loop corrections to the leading tanh-type term turn out to be small. They yield a broadening of the interface.
\end{abstract}

KEY WORDS: Interfaces, field theory

\section{Introduction}

Many systems of statistical physics can develop interfaces, separating different phases or substances. These include Bloch walls in ferromagnets, binary liquid mixtures, liquid-gas systems and systems of immiscible polymers [1, 2, 3, 4, 5. The properties of interfaces are important for the behaviour of such systems. They have been investigated both experimentally and theoretically and are subject of current research, see e.g. 6]. For example, if substrates are covered by liquid films in coatings or lubricants, the stability of the films depends on the interfacial tensions between substrate, liquid and vapor. Another example concerns the mechanical strength of polymer blends against deformations. The structure of interfaces between domains of the minority component, which are embedded in the background of the majority component, is of crucial importance for the strength of the blend 7 .

The profile of an interface characterizes its geometry. It determines several other properties such as the interface width and interface tension

*Institut für Theoretische Physik, Universität Münster, Wilhelm-Klemm-Str. 9, D48149 Münster, Germany; e-mail: munsteg@uni-muenster.de 
and therefore plays a central role for the physics of interfaces. Interfacial profiles are investigated experimentally through scattering of photons or neutrons 8 . Theoretical studies of interfacial profiles include numerical Monte Carlo simulations [9, 10, 11, 12] and field theoretic calculations [4.

The simplest description of interfaces is based on mean field theory [13]. The interfacial profile is described by a continuous order parameter $\phi(x)$ representing the difference between the concentrations of the two coexisting phases. In the Landau-Ginzburg approach the free energy density is written as 14 ]

$$
\mathcal{L}[\phi]=\frac{1}{2} \partial_{\mu} \phi \partial_{\mu} \phi+V_{0}(\phi) .
$$

In the situation with interfaces the potential is of the double-well type,

$$
V_{0}(\phi)=\frac{g_{0}}{4 !}\left(\phi^{2}-v_{0}^{2}\right)^{2} .
$$

The minima of this potential correspond to the two homogeneous phases. The mean field correlation length $\xi_{0}$ is defined through the second moment of the correlation function in the mean field approximation. It is given by the second derivative of the potential in its minima:

$$
\xi_{0}^{2}=\left(V_{0}^{\prime \prime}\left(v_{0}\right)\right)^{-1}=\frac{3}{g_{0} v_{0}^{2}} .
$$

In mean field theory the interfacial profile is given by minimization of the free energy density (11) with boundary conditions appropriate for an interface. This leads to the differential equation

$$
\Delta \phi-V_{0}^{\prime}(\phi)=0 .
$$

If we choose the interface to be perpendicular to the $z$-axis, we find the typical hyperbolic tangent profile [15.

$$
\phi(z)=v_{0} \tanh \left(\frac{z-z_{0}}{2 \xi_{0}}\right) .
$$

Its width is proportional to the mean field correlation length $\xi_{0}$. The parameter $z_{0}$ specifies the location of the interface and will be set to 0 in the following.

Corrections to mean field theory come from fluctuations of the order parameter field. They can be calculated systematically in renormalized perturbation theory. The fluctuations result in different modifications of the mean field result. First of all, higher order corrections change the form of the profile from the tanh-function to a different function $f$. Secondly, renormalization of the parameters $v_{0}$ and $\xi_{0}$ becomes necessary and consequently the mean field correlation length $\xi_{0}$ is replaced by the physical 
correlation length $\xi$, which diverges near the critical point with a characteristic exponent $\nu$. A profile of the form

$$
\phi(z)=v f(z / 2 \xi)
$$

corresponds to the scaling form proposed by Fisk and Widom [16]. Finally, long-wavelength capillary wave fluctuations lead to the roughening phenomenon [17. It implies a further broadening of the interface such that in a three-dimensional system the width of an interface depends logarithmically on the system size and diverges in the limit of an infinite system.

In this article we consider corrections to mean field theory by means of the "local potential approximation", which is popular in the study of non-homogeneous structures in field theory and cosmology. It takes into account corrections to the local potential $V(\phi)$, whereas contributions involving higher powers of derivatives are neglected. In this sense, the local potential approximation represents the leading contributions in a systematic expansion of the effective action. However, it neglects any non-local contribution to the effective action. Therefore it is, in particular, insensitive to effects related to the system size. The resulting interfacial profile is of the scaling form (6). In the local potential approximation the effects of capillary waves are not fully taken into account. This is due to the fact that in the differential equation determining the interface profile only the local value of the profile function but no large-scale properties like the system size enter. The profile function therefore describes a kind of intrinsic interfacial profile. Including large-scale effects would require to build on the full effective potential, which is a non-local functional of the profile function. In such a calculation additional contributions to the interface profile are to be expected, which depend logarithmically on the system size [17. To define a unique separation of an intrinsic profile from its capillary wave contribution in such a situation is a nontrivial subject, which has been discussed in various papers, see e.g. 1, 18, 4, 10, 12. In our approach, where broadening due to capillary waves is suppressed, this problem does not show up.

Our calculations are performed in $D=3$ physical dimensions in contrast to the $\epsilon$-expansions, where $D=4-\epsilon$ and an extrapolation to $\epsilon=1$ is necessary. The three-dimensional approach, in the framework of renormalized massive field theory, is based on a systematic expansion in a dimensionless coupling [19, 20]. We calculate the potential to second order in the loop expansion. Ultraviolet divergencies are treated by dimensional regularization $(D=3-\epsilon)$, which does not vitiate the fact that the results for physical quantities strictly refer to $D=3$ dimensions. Renormalization of the three-dimensional field theory is performed in the scheme used in [21] to two-loop order, employing the results of 22, 23. The differential equation for the interfacial profile is solved analytically in terms of special 
functions 24. The resulting profile is compared with results from Monte Carlo calculations.

Previous studies of interfacial profiles in the framework of field theory have been made in $[25,26,27,28$. In [25, 26, 28, the profile is calculated to first order in the $\epsilon$-expansion. This amounts to a one-loop calculation. As the expansion is around the four-dimensional case, capillary wave effects are not included, and an extrapolation to $D=3$ is necessary. The calculation of 25 is extended to include the effects of an external field in [28. In [27] the interfacial profile is considered in $D=3$ dimensions at one-loop order in the presence of an external gravitational field. A functional form of the profile is given, including capillary wave effects. The dependence on the system size is, however, not considered.

\section{Effective potential}

The effective potential can be calculated in the loop-expansion by standard field theoretic methods 29, 30, 31. The starting point is the Hamiltonian density

$$
h=\frac{1}{2} \partial_{\mu} \phi \partial_{\mu} \phi+V_{0}(\phi) .
$$

We write the mean field potential as

$$
V_{0}(\phi)=-\frac{m_{0}^{2}}{4} \phi^{2}+\frac{g_{0}}{4 !} \phi^{4}+\frac{3}{8} \frac{m_{0}^{4}}{g_{0}}=\frac{g_{0}}{4 !}\left(\phi^{2}-v_{0}^{2}\right)^{2},
$$

where the bare mass $m_{0}=1 / \xi_{0}$ is equal to the inverse bare (mean field) correlation length. The Hamiltonian is

$$
\mathcal{H}=\int d^{D} x h .
$$

In the physical situation the number of dimensions is of course $D=3$, but we shall keep $D$ variable to allow for dimensional regularization.

The normalized partition function with an external source is defined through the functional integral

$$
Z[j]=\frac{1}{Z_{0}} \int \mathcal{D} \phi(x) \exp \left\{-\mathcal{H}[\phi]+\int d^{D} x j(x) \phi(x)\right\},
$$

where

$$
Z_{0}=\int \mathcal{D} \phi(x) \exp \{-\mathcal{H}[\phi]\},
$$

and the usual prefactor $\beta=1 / k T$ is set to 1 by a suitable normalization. The free energy is

$$
W[j]=\ln Z[j],
$$


and finally the Gibbs potential, which is called effective action in field theory, is obtained by a Legendre transformation

$$
\Gamma\left[\phi_{c}\right]=W[j]-\int d^{D} x j(x) \phi_{c}(x),
$$

where the so-called "classical field" is

$$
\phi_{c}(x) \doteq \frac{\delta W[j]}{\delta j(x)} .
$$

For a constant function $\phi_{c}(x) \equiv \phi$ the effective action yields the effective potential $V(\phi)$ via

$$
-\Gamma[\phi]=\int d^{D} x V(\phi) .
$$

More generally, in a derivative expansion the effective action can be represented as

$$
-\Gamma[\phi]=\int d^{D} x\left\{V(\phi(x))+\frac{1}{2} \mathcal{Z}(\phi(x))(\partial \phi(x))^{2}+\cdots\right\},
$$

where the dots imply terms with a higher number of derivatives, and $\mathcal{Z}(\phi(x))$ is a field renormalization factor.

The effective potential $V(\phi)$ is the basic quantity for the discussion of spontaneous symmetry breaking and non-homogeneous configurations 32 . It should be noted that it is not a convex function, as the loop expansion also reveals. This is, however, not a failure of the loop expansion, but a feature of the effective potential, see e.g. [33, 34] or sec. 2.2 of the textbook [35. The local potential represents the density of the Gibbs potential for a small volume element, in which the field is constant, i.e. locally in a pure phase. The proof of convexity applies to the extensive thermodynamic function, i.e. the total Gibbs potential, and not to the local potential. For a proof of convexity in the context of field theory see [36, 30. In a situation, where the local potential is not convex, but the Gibbs potential is, the system is in a mixed phase and the convexity of the total Gibbs potential is the result of the composition of contributions from the coexisting pure phases. This is the situation where interfaces are present. Therefore it is the local effective potential which is relevant for the local interface structure. Another non-homogeneous situation, where the non-convex local potential plays a role, is the presence of nucleation bubbles in nucleation theory [37, 38 .

The effective action can be calculated by means of the loop expansion in the form

$$
\Gamma[\phi]=\Gamma_{0}[\phi]+\Gamma_{1}[\phi]+\Gamma_{2}[\phi]+\cdots,
$$


where the leading term $\Gamma_{0}=-\mathcal{H}$ is equal to the negative Hamiltonian. In the corresponding expansion of the effective potential,

$$
V(\phi)=V_{0}(\phi)+V_{1}(\phi)+V_{2}(\phi)+\cdots,
$$

the leading term $V_{0}$ is the mean field potential (8).

The first order contribution to the effective action is

$$
\Gamma_{1}[\phi]=-\frac{1}{2} \operatorname{Tr} \ln \frac{K(\phi)}{K(0)}
$$

with the differential operator

$$
K(\phi)=-\Delta-\frac{m_{0}^{2}}{2}+\frac{g_{0}}{2} \phi^{2} .
$$

Setting $\phi$ constant, the first order correction to the effective potential can be obtained by Fourier transform as

$$
\begin{aligned}
V_{1}(\phi) & =\frac{1}{2} \int \frac{d^{D} k}{(2 \pi)^{D}} \ln \left(1+\frac{g_{0} \phi^{2} / 2}{k^{2}-m_{0}^{2} / 2}\right) \\
& =\frac{1}{12 \pi}\left\{m_{0}^{3}-\left(-\frac{m_{0}^{2}}{2}+\frac{g_{0}}{2} \phi^{2}\right)^{\frac{3}{2}}\right\} .
\end{aligned}
$$

For small values of the field, $|\phi|<\sqrt{m_{0}^{2} / g_{0}}$, this contribution has an imaginary part. Actually, for small values of the field the system is unstable due to spinodal decomposition. The boundary value for $|\phi|$ given above represents the mean field spinodal. In the full renormalized theory this will be modified or even be replaced by a crossover line 39. According to 33. the imaginary part of the effective potential is to be identified with the decay rate of the mixed state. For the calculation of the interfacial profile the real part of the effective potential has to be employed.

The two-loop contribution to the effective potential is obtained through the calculation of two Feynman diagrams:

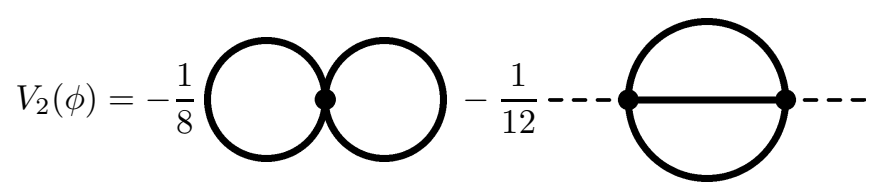

In the two-loop contribution usual ultraviolet divergencies appear. They originate from large momentum, short-distance fluctuations, and are not related to capillary wave fluctuations. This is reflected in the fact that they 
are identical to those coming from bulk fluctuations in a situation without interfaces.

The divergencies have to be treated in some regularization and renormalization scheme. We choose to employ dimensional regularization in $D=3-\epsilon$ dimensions. It should be noted that this does not amount to an $\epsilon$-expansion, since after renormalization $\epsilon$ is sent to zero, whereas in the $\epsilon$-expansion one has $D=4-\epsilon$ and the results have to be extrapolated to $\epsilon=1$. We obtain

$$
\begin{aligned}
& V_{2}(\phi)=-\frac{g_{0}}{8 \pi} \frac{m_{0}^{2}}{32 \pi} \\
& +\left(\frac{g_{0}}{8 \pi}\right)^{2} m_{0}^{-2 \epsilon} \frac{\phi^{2}}{6}\left\{-\frac{1}{\epsilon}+\frac{1}{2}+\gamma+\ln \left(\frac{9}{4 \pi}\right)+\ln \left(-\frac{1}{2}+\frac{g_{0} \phi^{2}}{2 m_{0}^{2}}\right)\right\},
\end{aligned}
$$

where $\gamma=0.577 \ldots$ is Euler's constant. This potential is being used for the calculation of the interfacial profile.

\section{The Profile}

In the local potential approximation the effective action is approximated by

$$
-\Gamma_{\mathrm{LPA}}[\phi]=\int d^{D} x\left\{V(\phi(x))+\frac{1}{2 Z_{3}}(\partial \phi(x))^{2}\right\} .
$$

The factor $Z_{3}$ is the usual field renormalization constant in the broken symmetry phase, given by

$$
Z_{3}=\frac{1}{\mathcal{Z}(v)}
$$

with $v$ denoting the minimum of the effective potential.

The interfacial profile $\phi(z)$ is now obtained in the local potential approximation as a solution of the differential equation

$$
Z_{3}^{-1} \Delta \phi-V_{r}^{\prime}(\phi)=0,
$$

where $V_{r}$ denotes the real part of the effective potential. For an interface perpendicular to the $z$-axis the appropriate boundary conditions are

$$
\begin{aligned}
\lim _{z \rightarrow \infty} \phi(z) & =v \\
\lim _{z \rightarrow-\infty} \phi(z) & =-v .
\end{aligned}
$$

The resulting profile function contains diverging coefficients coming from the $1 / \epsilon$ term in the second order effective potential (23). These are treated by the usual renormalization procedure. We adopt the renormalization scheme used in 21] to two-loop order. The renormalized mass 
$m_{R}=1 / \xi$ is equal to the inverse correlation length $\xi$, which in turn is defined through the second moment of the correlation function. The field $\phi$ and its value $v$ in the minimum are renormalized according to

$$
\phi_{R}(x)=\frac{1}{\sqrt{Z_{3}}} \phi(x), \quad v_{R}=\frac{1}{\sqrt{Z_{3}}} v .
$$

The renormalized coupling is specified as in 40, through

$$
g_{R}=\frac{3 m_{R}^{2}}{v_{R}^{2}} .
$$

In addition we define a dimensionless renormalized coupling according to

$$
u_{R}=\frac{g_{R}}{m_{R}^{4-D}} .
$$

Employing the relations given in 22, 23], the bare quantities $m_{0}$ and $g_{0}$ are expressed in terms of their renormalized counterparts. This yields an expression for the renormalized interfacial profile $\phi_{R}(z)$ depending on the parameters $m_{R}$ and $u_{R}$. In this expression the divergencies are canceled, as they should.

The solution is expanded in the form

$$
\begin{aligned}
\phi_{R}(z) & =\sqrt{\frac{3 m_{R}}{u_{R}}}\left\{\chi_{0}(\hat{z})+\frac{u_{R}}{8 \pi} \chi_{1}(\hat{z})+\left(\frac{u_{R}}{8 \pi}\right)^{2} \chi_{2}(\hat{z})+\mathcal{O}\left(u_{R}^{3}\right)\right\} \\
& \equiv \sqrt{\frac{3 m_{R}}{u_{R}}} \chi(\hat{z}) .
\end{aligned}
$$

Here we have introduced the variable

$$
\hat{z}=\frac{m_{R}}{2} z .
$$

The leading order contribution has of course the form of the mean field profile

$$
\chi_{0}(\hat{z})=\tanh \hat{z} .
$$

It should, however, be distinguished from the mean field profile, as it contains the physical correlation length instead of the mean field one. It represents a refinement of Landau theory that is consistent with scaling [16] and holds near the critical point.

Due to the fact that the effective potential has a point of non-analyticity, the first and second order contributions are also non-analytic. For $|\hat{z}|$ smaller or larger than $\operatorname{artanh}(1 / \sqrt{3})$, the functions $\chi_{1}$ and $\chi_{2}$ take different forms, which are called $\chi_{1 s}, \chi_{2 s}$ and $\chi_{1 l}, \chi_{2 l}$, respectively. For the first and second order correction we obtain [24]

$$
\chi_{1 s}(\hat{z})=\frac{1}{12} \hat{z} \operatorname{sech}^{2} \hat{z}+\frac{2}{9} \sinh \hat{z} \cosh \hat{z}-\frac{2}{3} \tanh \hat{z}
$$




$$
\begin{aligned}
\chi_{1 l}(\hat{z})= & \frac{1}{12} \hat{z} \operatorname{sech}^{2} \hat{z}-\frac{1}{12} \operatorname{sech}^{2} \hat{z} \operatorname{artanh}\left(\frac{\sqrt{-\frac{1}{2}+\sinh ^{2} \hat{z}}}{\sinh \hat{z}}\right) \\
& +\frac{2}{9} \sinh \hat{z} \cosh \hat{z}-\frac{2}{3} \tanh \hat{z} \\
& -\sinh \hat{z} \sqrt{-\frac{1}{2}+\sinh ^{2} \hat{z}}\left(\frac{2}{9}-\frac{1}{2} \operatorname{sech}^{2} \hat{z}\right),
\end{aligned}
$$

and

$$
\begin{aligned}
\chi_{2 s}(\hat{z})= & -\frac{1}{144} \hat{z}^{2} \operatorname{sech}^{2} \hat{z} \tanh \hat{z} \\
& +\hat{z}\left(-\frac{13}{216}+\frac{17}{216} \cosh ^{2} \hat{z}-\frac{1}{24} \sinh ^{2} \hat{z}-\frac{815}{1728} \operatorname{sech}^{2} \hat{z}\right) \\
& +\frac{343}{648} \cosh \hat{z} \sinh \hat{z}+\frac{2}{81} \cosh ^{3} \hat{z} \sinh \hat{z}-\frac{217}{216} \tanh \hat{z} \\
& +\frac{1}{6} \operatorname{sech}^{2} \hat{z}\left(\sqrt{3}-\operatorname{artanh} \frac{1}{\sqrt{3}}\right) \operatorname{artanh}(\sqrt{3} \tanh \hat{z}) \\
& +\frac{1}{48} \operatorname{sech}^{2} \hat{z}(\sinh (4 \hat{z})-4 \hat{z}) \ln \left(\frac{1}{2}-\frac{3}{2} \tanh ^{2} \hat{z}\right) \\
& +\frac{1}{24} \operatorname{sech}^{2} \hat{z}\left\{\operatorname{Li}_{2}\left(\frac{1-\sqrt{3} \tanh \hat{z}}{1-\sqrt{3}}\right)-\operatorname{Li}_{2}\left(\frac{1-\sqrt{3} \tanh \hat{z}}{1+\sqrt{3}}\right)\right. \\
& \left.\quad-\operatorname{Li}_{2}\left(\frac{1+\sqrt{3} \tanh ^{\hat{z}}}{1-\sqrt{3}}\right)+\operatorname{Li}_{2}\left(\frac{1+\sqrt{3} \tanh \hat{z}}{1+\sqrt{3}}\right)\right\} \\
\chi_{2 l}(\hat{z})= & \left.\frac{11 \sqrt{3}}{108}-\frac{37}{108} \operatorname{artanh} \frac{1}{\sqrt{3}}\right) \operatorname{sech}^{2} \hat{z} \operatorname{sgn}^{2} \\
& +\frac{1}{144} \operatorname{sech}^{2} \hat{z} \tanh \hat{z} \operatorname{artanh}^{2}\left(\frac{\sqrt{-\frac{1}{2}+\sinh ^{2} \hat{z}}}{\sinh _{\hat{z}}}\right) \\
& \left.+\frac{1}{108} \operatorname{artanh}^{2} \frac{\sqrt{-\frac{1}{2}+\sinh ^{2} \hat{z}}}{\sinh \hat{z}}\right)\left\{2-4 \cosh ^{2} \hat{z}+\frac{223}{16} \operatorname{sech}^{2} \hat{z} \tanh \hat{z}\right. \\
& \left.+\left(4 \cosh \hat{z}+\operatorname{sech} \hat{z}-9 \operatorname{sech}^{3} \hat{z}\right) \sqrt{-\frac{1}{2}+\sinh ^{2} \hat{z}}\right\}
\end{aligned}
$$




$$
\begin{aligned}
& +\sqrt{-\frac{1}{2}+\sinh ^{2} \hat{z}}\left\{\frac{1}{108} \hat{z}\left(-4 \cosh \hat{z}-\operatorname{sech} \hat{z}+9 \operatorname{sech}^{3} \hat{z}\right)\right. \\
& \left.\quad-\frac{233}{648} \sinh \hat{z}-\frac{4}{81} \cosh ^{2} \hat{z} \sinh \hat{z}+\frac{145}{288} \operatorname{sech} \hat{z} \tanh \hat{z}\right\} \\
& -\frac{1}{144} \hat{z}^{2} \operatorname{sech}^{2} \hat{z} \tanh \hat{z} \\
& +\hat{z}\left(-\frac{13}{216}+\frac{17}{216} \cosh ^{2} \hat{z}-\frac{1}{24} \sinh ^{2} \hat{z}-\frac{223}{1728} \operatorname{sech}^{2} \hat{z}\right) \\
& +\frac{209}{648} \cosh \hat{z} \sinh \hat{z}+\frac{4}{81} \cosh ^{3} \hat{z} \sinh \hat{z} \\
& -\frac{19}{27} \tanh ^{\hat{z}}-\frac{3}{8} \operatorname{sech} \hat{z} \tanh \hat{z} \\
& +\frac{1}{6} \operatorname{sech}^{2} \hat{z}\left(\sqrt{3}-\operatorname{artanh}^{2}\right) \operatorname{artanh}\left(\frac{1}{\sqrt{3} \tanh _{\hat{z}}}\right) \\
& +\frac{1}{48} \operatorname{sech}^{2} \hat{z}(\sinh (4 \hat{z})-4 \hat{z}) \ln \left(-\frac{1}{2}+\frac{3}{2} \tanh \hat{z}\right) \\
& +\frac{1}{24} \operatorname{sech}^{2} \hat{z}\left\{\operatorname{Li}_{2}\left(\frac{1-\sqrt{3} \tanh \hat{z}}{1-\sqrt{3}}\right)-\operatorname{Li}_{2}\left(\frac{1-\sqrt{3} \tanh \hat{z}}{1+\sqrt{3}}\right)\right. \\
& \left.\quad-\operatorname{Li}_{2}\left(\frac{1+\sqrt{3} \tanh \hat{z}}{1-\sqrt{3}}\right)+\operatorname{Li}_{2}\left(\frac{1+\sqrt{3} \tanh \hat{z}}{1+\sqrt{3}}\right)\right\}
\end{aligned}
$$

The contributions of the various orders and the resulting profile function are displayed in Fig.1. For the renormalized dimensionless coupling we have chosen $u_{R}=14.3$. In the vicinity of the critical point the coupling varies only slowly and is close to the universal fixed point value $u_{R}^{*}=$ 14.3(1), see 41] for a discussion of numerical and field-theoretical estimates.

The figure shows that the corrections are small compared to the leading term and therefore the interfacial profile at second order can be approximately described by a tanh function. It can also be seen that the higher order corrections lead to an increase of the interface width. The broadening of the interface can be specified more quantitatively in terms of a broadening factor $\alpha$ by fitting the total profile function to

$$
\chi_{\text {fit }}(\hat{z})=\left(1-\frac{1}{6}\left(\frac{u_{R}}{8 \pi}\right)^{2}\right) \tanh (\alpha \hat{z})
$$

where the prefactor is fixed through the asymptotic behaviour of $\chi(\hat{z})$. For $u_{R}=14.3$ we obtain a value of $\alpha=0.80$.

While the one-loop contribution $\chi_{1}$ vanishes asymptotically for $|z| \rightarrow$ $\infty$, the two-loop contribution approaches a small but finite value, affecting 


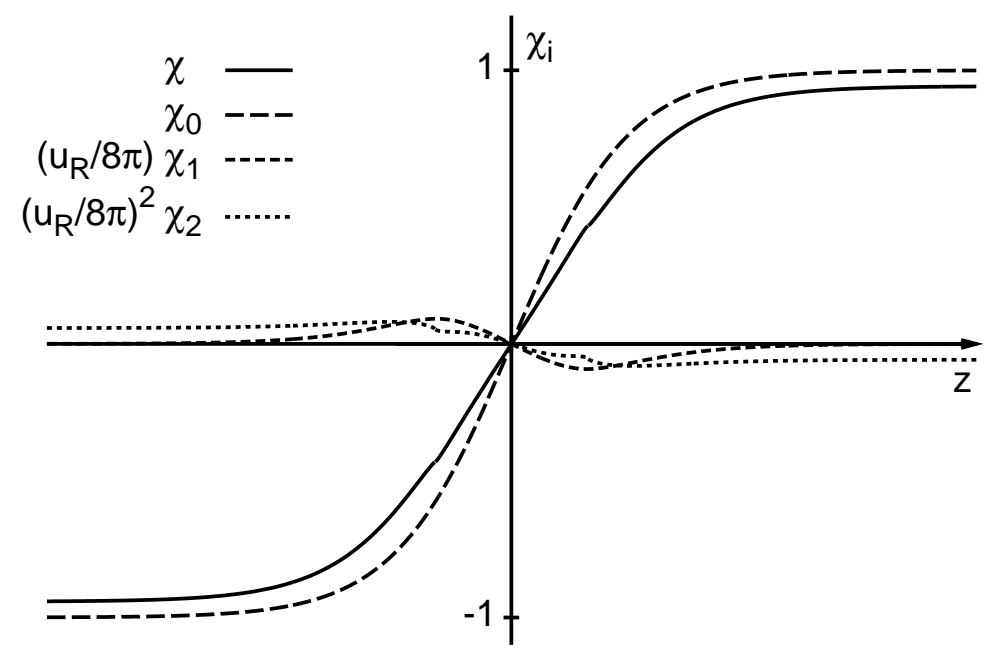

Figure 1: The renormalized interfacial profile to second order. The curves represent the contributions of the different orders and their sum for the choice $u_{R}=14.3$.

the total asymptotic value. It also displays a bump-like behaviour, which is due to the non-analyticity at $\operatorname{artanh}(1 / \sqrt{3})$, mentioned above.

A numerical investigation of the interfacial profile in the three-dimensional Ising model has been made by Stauffer [9] for a temperature, which is one percent below the critical one. The resulting order parameter can be described rather well by a tanh function and the numerical fit yields

$$
\phi=0.372 \tanh (0.1 z / a),
$$

where $a$ denotes the lattice spacing.

In order to compare with our result we have to convert the units by using

$$
m_{R}=\frac{1}{\xi}=\frac{1}{a f_{-}}\left|\frac{T-T_{c}}{T_{c}}\right|^{\nu}
$$

with $f_{-}=0.2502(8)$ 42] and $\nu=0.630$ [43, 44]. For $T / T_{c}=0.99$ this gives

$$
0.80 \frac{m_{R}}{2} z=0.088 \frac{z}{a} \text {. }
$$

The broadening factor deviates from the Monte Carlo value by only $12 \%$. The significance of this coincidence is, however, not clear, due to 
the procedure to identify the interface profile in the Monte Carlo calculation. This procedure includes the effects of capillary waves, but neglects the effects of bulk fluctuations, whose contributions are central in our calculation. It is unclear how the resulting difference in the definitions of the interface width affects the comparison.

\section{Conclusion}

We investigated the interfacial profile in the framework of renormalized field theory using the local potential approximation. For this purpose the effective potential has been calculated in the loop expansion to second order. The resulting profile function is obtained analytically. It is of the scaling form and can be interpreted as an intrinsic profile. To lowest order the profile is of the mean field type. For typical values of the coupling constant in the scaling region the higher order corrections are small. They imply a broadening of the interface of about $25 \%$.

\section{References}

[1] B. Widom, in Phase Transitions and Critical Phenomena, Vol. 2, C. Domb and M. Green, eds. (Academic Press, New York, 1972).

[2] J. Rowlinson and S. Widom, Molecular Theory of Capillarity (Clarendon Press, Oxford, 1982).

[3] K. Binder, Critical behaviour at surfaces, in Phase Transitions and Critical Phenomena, Vol. 8, C. Domb and J. Lebowitz, eds. (Academic Press, New York, 1983).

[4] D. Jasnow, Critical phenomena at interfaces, Rep. Prog. Phys. 47: 1059-1132 (1984).

[5] S. A. Safran, Statistical Thermodynamics of Surfaces, Interfaces, and Membranes (Addison-Wesley, Reading, 1994).

[6] K. Binder, Finite size effects in thin film simulations, in [11, (2003).

[7] K. Binder, Simulations of interfaces between coexisting phases: multiscale aspects, in Multiscale Simulations in Chemistry and Physics, A. Brandt, J. Bernholc and K. Binder, eds. (IOS Press, Amsterdam, 2001).

[8] J. S. Huang and W. W. Webb, Viscous damping of thermal excitations on the interface of critical fluid mixtures, Phys. Rev. Lett. 23: 160163 (1969); D. Langevin, ed., Light Scattering by Liquid Surfaces and 
Complementary Techniques (Dekker, New York, 1992); R. K. Thomas, Neutron reflection from liquid interfaces, Annual Review of Physical Chemistry 55: 91-426 (2004).

[9] D. Stauffer, Oil-water interfaces in the Ising-model, Progr. Colloid Polym. Sci. 103: 60-66 (1997).

[10] A. Werner, F. Schmid, M. Müller and K. Binder, Anomalous sizedependence of interfacial profiles between coexisting phases of polymer mixtures in thin film geometry: A Monte-Carlo simulation, J. Chem. Phys. 107: 8175-8188 (1997); A. Werner, F. Schmid, M. Müller and K. Binder, "Intrinsic" profiles and capillary waves at homopolymer interfaces: A Monte Carlo study, Phys. Rev. E 59: 728-738 (1999).

[11] B. Dünweg, D. P. Landau and A. Milchev, eds., Computer Simulations of Surfaces and Interfaces (Kluwer Acad. Publ., Dordrecht, 2003).

[12] M. Müller and G. Münster, Profile and width of rough interfaces, $J$. Stat. Phys. 118: 669-686 (2005).

[13] J. van der Waals, The thermodynamic theory of capillarity under the hypothesis of a continuous variation of density, Verhandel Konink. Akad. Weten. 1 (1893). English translation: J. Rowlinson, J. Stat. Phys. 20: 97-244 (1979).

[14] M. Le Bellac, Quantum and Statistical Field Theory (Clarendon Press, Oxford, 1991).

[15] J. Cahn and J. Hilliard, Free energy of a nonuniform system, J. Chem. Phys. 28: 258-267 (1958).

[16] S. Fisk and B. Widom, Structure and free energy of the interface between fluid phases in equilibrium near the critical point, J. Chem. Phys. 50: 3219-3227 (1960).

[17] F. Buff, R. Lovett and F. Stillinger, Interfacial density profile for fluids in the critical region, Phys. Rev. Lett. 15: 621-623 (1965).

[18] J. Weeks, Structure and thermodynamics of the liquid-vapor interface, J. Chem. Phys. 67: 3106-3121 (1977).

[19] G. Parisi, Field-theoretic approach to second-order phase transition in two-and three-dimensional systems, J. Stat. Phys. 23: 49 - 82 (1980).

[20] J. C. Le Guillou and J. Zinn-Justin, Critical exponents from field theory, Phys. Rev. B 21: 3976 - 3998 (1980). 
[21] G. Münster, Interface tension in three-dimensional systems from field theory, Nucl. Phys. B 340: 559-567 (1990).

[22] G. Münster and J. Heitger, Field-theoretic calculation of the universal amplitude ratio of correlation lengths in $3 D$ Ising systems, Nucl. Phys. B 424: 582-594 (1994).

[23] C. Gutsfeld, J. Küster and G. Münster, Calculation of universal amplitude ratios in three-loop order, Nucl. Phys. B 479: 654-662 (1996).

[24] J. Küster, Kritisches Verhalten fluktuierender Grenzflächen, $\mathrm{PhD}$ thesis, Univ. of Münster, 2001.

[25] T. Ohta and K. Kawasaki, Renormalization group approach to the interfacial order parameter profile near the critical point, Prog. Theor. Phys. 58: 467-481 (1977).

[26] J. Rudnick and D. Jasnow, $\epsilon$ expansion for the interfacial profile, Phys. Rev. B 17: 1351-1354 1978.

[27] D. Jasnow and J. Rudnick, Interfacial profile in three Dimensions, Phys. Rev. Lett. 41: 698-701 (1978).

[28] J. H. Sikkenk and J. M. J. van Leeuwen, $\epsilon$-expansion for the interfacial profile in an external field, Physica A 137: 156-177 (1986).

[29] R. Jackiw, Functional evaluation of the effective potential, Phys. Rev. D 9: 1686-1701 (1974).

[30] J. Iliopoulos, C. Itzykson and A. Martin, Functional methods and perturbation theory, Rev. Mod. Phys. 47: 165-192 (1975).

[31] D. J. Amit, Field Theory, the Renormalization Group and Critical Phenomena (McGraw-Hill, New York, 1978).

[32] S. Coleman and E. Weinberg, Radiative corrections as the origin of spontaneous symmetry breaking, Phys. Rev. D 7: 1888-1910 (1973).

[33] E. J. Weinberg and A. Wu, Understanding complex perturbative effective potentials, Phys. Rev. D 36: 2474-2480 (1987).

[34] M. Sher, Electroweak Higgs potentials and vacuum stability, Phys. Reports 179: 273-418 (1989).

[35] H. Meyer-Ortmanns and T. Reisz, Principles of Phase Structures in Particle Physics (World Scientific, Singapore, 2007). 
[36] K. Symanzik, Renormalizable models with simple symmetry breaking. I. Symmetry breaking by a source term, Commun. Math. Phys. 16: 48-80 (1970).

[37] J. S. Langer, Statistical theory of the decay of metastable states, Ann. Phys. 54: 258-275 (1969).

[38] G. Münster and S. Rotsch, Analytical calculation of the nucleation rate for first order phase transitions beyond the thin wall approximation, Eur. Phys. J. C 12: 161-171 (2000).

[39] K. Binder, Spinodal decomposition, in Materials Science and Technology Vol. 5, Phase Transformations in Materials, R. W. Cahn, P. Haasen and E. J. Kramer, eds. (Wiley-VCH, Weinheim, 1991).

[40] M. Lüscher and P. Weisz, Scaling laws and triviality bounds in the lattice $\phi^{4}$-theory; one-component model in the phase with spontaneous broken symmetry, Nucl. Phys. B 295: 65-92 (1987).

[41] M. Caselle and M. Hasenbusch, Universal amplitude ratios in the 3D Ising model, J. Phys. A 30: 4963-4982 (1997).

[42] A. J. Liu and M. E. Fisher, The three-dimensional Ising model revisited numerically, Physica A 156: 35-76 (1989).

[43] R. Guida and J. Zinn-Justin, Critical exponents of the N-vector model, J. Phys. A 31: 8103-8121 (1998).

[44] M. Hasenbusch, K. Pinn and S. Vinti, Critical exponents of the 3D Ising universality class from finite size scaling with standard and improved actions, Phys. Rev. B59: 11471 (1999). 